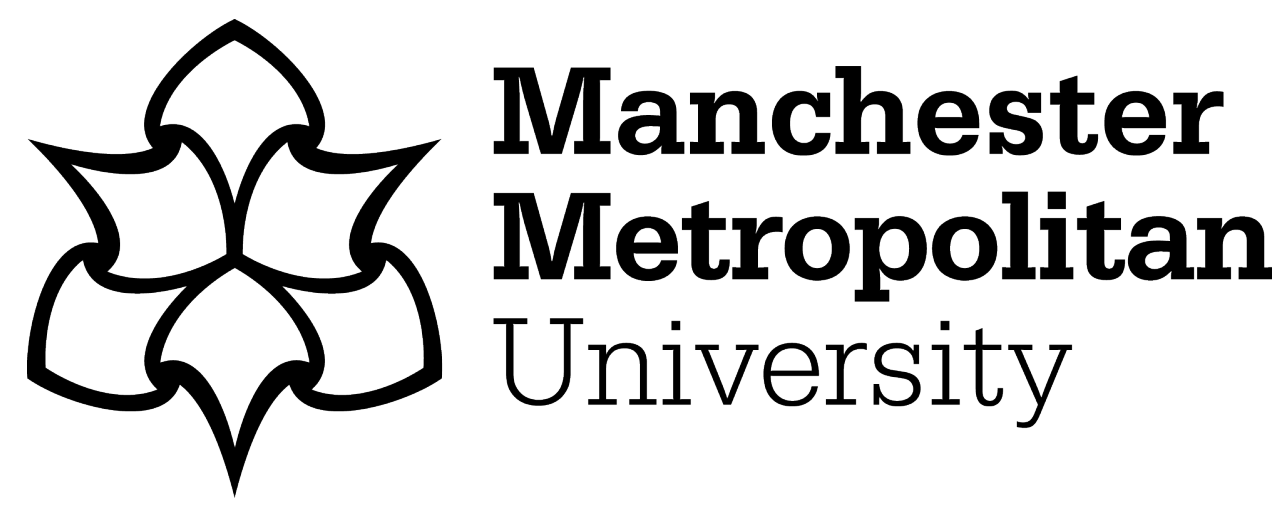

Zahnow, R, McVeigh, J ORCID logoORCID: https://orcid.org/0000-00015319-6885, Bates, G, Hope, V, Kean, J, Campbell, J and Smith, J (2018) Identifying a typology of men who use Anabolic Androgenic Steroids (AAS). International Journal of Drug Policy, 55. pp. 105-112. ISSN 0955-3959

Downloaded from: https://e-space.mmu.ac.uk/624829/

Version: Accepted Version

Publisher: Elsevier

DOI: https://doi.org/10.1016/j.drugpo.2018.02.022

Usage rights: Creative Commons: Attribution-Noncommercial-No Derivative Works 4.0

Please cite the published version 


\title{
Identifying a typology of men who use Anabolic Androgenic Steroids (AAS)
}

\author{
Renee Zahnow ${ }^{1 *}$ \\ Jim McVeigh ${ }^{2}$ \\ (J.McVeigh@ljmu.ac.uk) \\ Geoff Bates ${ }^{2}$ \\ (G.P.Bates@2016.ljmu.ac.uk) \\ Vivian Hope ${ }^{2}$ \\ (V.D.Hope@ljmu.ac.uk) \\ Joseph Kean ${ }^{2}$ \\ (joseph@ninezerofive.org) \\ Josie Smith ${ }^{3}$ \\ (Josie.Smith@wales.nhs.uk) \\ John Campbel1 ${ }^{4}$ \\ (John.Campbell@ggc.scot.nhs.uk)
}

1 The Institute for Social Science Research, The University of Queensland, 80 Meiers Rd, Indooroopilly, Brisbane, Queensland, Australia 4068.

2Public Health Institute, Liverpool John Moore's University, Henry Cotton Campus, 15-21 Webster Street, Liverpool, L3 2ET.

3Public Health Wales, Health Promotion, Cardiff, United Kingdom.

${ }^{4}$ Research, Development and Innovation at Scottish National Blood Transfusion Service, Edinburgh, United Kingdom.

Funding: This work was supported by Public Health Wales as part of the annual IPED research programme at Liverpool John Moore's University Public Health Institute.

Conflicts of interest: There are no conflicts of interest.

*Corresponding Author

Renee Zahnow

Address: The University of Queensland

Institute for Social Science Research

St Lucia QLD 4072

Phone number: +61402204381

Email: r.zahnow@uq.edu.au 
WORD COUNT: 3415 (not including reference list or tables) 


\section{Identifying a typology of men who use Anabolic Androgenic Steroids (AAS)}

\section{Abstract}

Background: Despite recognition that the Anabolic Androgenic Steroid (AAS) using population is diverse, scholarly attempts to develop theories to conceptualise this variance in use have been limited.

Methods: In this study, using cluster analysis and multinomial logistic regression, we identify typologies of AAS users and examine variations in motivations for AAS use across types in a sample of 611 men who use AAS.

Results: The cluster analysis identified four groups in the data with different risk profiles. These groups largely reflect the ideal types of AAS users proposed by Christiansen and colleagues (2016): Cluster 1 (You Only Live Once $(Y O L O)$ type, $\mathrm{n}=68,11.1 \%$ ) were younger and motivated by fat loss; Cluster 2 (Well-being type, $n=236,38.6 \%$ ) were concerned with getting fit; Cluster 3 (Athlete type, $n=155,25.4 \%$ ) were motivated by muscle and strength gains; Cluster 4 (Expert type, $n=152,24.9 \%$ ) were focused on specific goals (i.e. not 'getting fit').

Conclusion: The results of this study demonstrate the need to make information about AAS accessible to the general population and to inform health service providers about variations in motivations and associated risk behaviours. Attention should also be given to ensuring existing harm minimisation services are equipped to disseminate information about safe intra-muscular injecting and ensuring needle disposal sites are accessible to different user groups.

Keywords: anabolic steroids; image and performance enhancing drugs; bodybuilding; harm minimisation; risk 


\section{Identifying a typology of men who use Anabolic Androgenic Steroids (AAS)}

\section{Introduction}

The use of anabolic androgenic steroids (AAS) for muscular gain, performance and image enhancement is not new. The use of AAS for athletic purposes was first noted among the United States bodybuilding community in the 1950s and soon after appeared in other sports (Kanayama \& Pope Jr, 2017). Use of AAS was largely restricted to the elite sporting community until the 1980s when images of Arnold Schwarzenegger in Conan the Barbarian (following his mainstream media launch in the bodybuilding cult classic Pumping Iron) and Sylvester Stallone in Rambo and the Rocky series, among others, propelled the bodybuilder physique into mainstream idealised depictions of the male body. AAS use among recreational gym goers and non-athletes wanting to gain muscle and strength increased over the ensuing decades. Most recently, technological advances have resulted in new ways of gaining information and discussing the use of AAS and associated drugs. This may account, in some ways, for the diversity of motivations and patterns of AAS use observed in the contemporary AAS using population; social media and online forums have provided new ways of sharing information about the use of substances and displaying their effects on the body. The growth of the internet and developments in global transportation combined with low cost manufacturing has increased availability, ease of access and affordability of these drugs \{Brennan, Wells and Van Hout, 2017\} (Evans-Brown, Kimergård, \& McVeigh, 2009; McVeigh, Evans-Brown, \& Bellis, 2012).

While household surveys suggest lifetime prevalence of AAS use has remained relatively low and stable since the 1990s, at least in western world countries, there is agreement among scholars that the use of steroids is a growing public health issue \{Vinther, 2015\}. Statistics demonstrate that the number of individuals accessing needle and syringe programs (NSPs) for steroid injection equipment has grown substantially in recent years; a pattern that is evident internationally in countries including Australia (Jacka et al 2017), the UK \{McVeigh and Bagley, 2016\} and USA \{Rich et al., 1999\}. In the UK, the number of syringes dispensed per individual has also increased over the last decade \{Vinther, 2015\}. While these statistics should not be interpreted as evidence of an escalation in the prevalence of steroid use, they 
highlight the need to consider the capacity of current drug services to address the needs of steroid users and to develop initiatives specifically targeted toward harm minimisation for this group. In order to develop these initiatives it is necessary to identify typical patterns of steroid use and prominent risk behaviours.

Given the heterogeneity evident in steroid use, attributable to the complexity of drug regimens, high levels of polypharmacy and variation in training goals and motivations, it is exceedingly difficult to describe the 'typical' user or pattern of use. Indeed, evidence suggests the possibility of multiple subgroups of steroid users with different patterns of drug use and related risks \{Evans, 1997\}\{Hildebrandt, Langenbucher, Carr and Sanjuan 2007\} \{Wilkinson, 1987\}. The use of typologies to identify distinct subgroups within drug using populations is not new. This approach has been applied within groups of cocaine \{Schonnesson, 2008 ; opioid \{Bennett et al., 2017\}\{Florez et al., 2015\} and alcohol users \{Peacock et al 2016\}. These studies employ empirical classification techniques (e.g. cluster analysis; latent class analysis) to address issues associated with the multidimensionality of drug use by grouping individuals who use substances into 'types' based on core defining features that characterise their drug use and influence risk and required intervention. Empirical classification techniques quantitatively identify relatively homogenous subgroups within heterogenous samples \{Clatworthy et al., 2005\}. These techniques can capture multiple variables and simultaneously consider user characteristics (age, gender, ethnicity), patterns of drug use (frequency, dosage and administration), abuse severity and effects (physical, social and psychological) and/or motivations for use $\{$ Bennett et al 2017\}. They identify certain 'sets' of characteristics and behaviours that tend to co-occur and can be used to inform interventions that are tailored to the risk profile of different 'types' of drug users. For example, latent class analysis was employed by Bennett and colleagues $\{$ Bennett 2017$\}$ to identify common combinations of behaviours among opioid users that were associated with greater risk of overdose. Similarly, Florez and colleagues \{Florez 2015\} applied cluster analysis to classify new opioid users seeking treatment and better understand treatment service demands in Spain.

To date, only one study has applied this type of analysis to steroid use. Hildebrandt, Langenbucher, Carr and 
Sanjuan \{Hildebrandt et al 2007\} employed latent class analysis, latent trait analysis and factor mixture models to examine patterns of steroid use in a sample of 400 men recruited through internet discussion forums. They found evidence to support the existence of a four-class factor mixture model; inter-group distinctions were related to combinations of substances and training goals \{Hildebrandt et al 2007\}. Each class was associated with different levels of risk. Class $1(10.75 \%)$ was associated with the greatest levels of risk. This group engaged in high levels of polypharmacy and used a range of steroids in addition to various licit and illicit IPEDs. Class $2(16.75 \%)$ were primarily motivated by fat burning and had the greatest probability of using illicit thermogenics and stanozolol (a steroid used to maintain leanness). Class 3 (20.75\%) was primarily concerned with muscle building and tended to use steroids associated with adding muscle mass. Class $4(51.75 \%)$ demonstrated the lowest levels of risk and was referred to as the normative group. This was the largest group and was characterised by the use of common forms of steroids (testosterone, methandrostenolone) along with legal, over the counter supplements associated with fat burning \{Hildebrandt et al 2007\}.

Other efforts to distinguish distinct subpopulations of steroid users have adopted a qualitative approach and categorised individuals who use steroids according to primary motivation for use (Christiansen et al., 2016; Hakansson, Mickelsson, Wallin, \& Berglund, 2012; Hanley Santos \& Coomber, 2017; Kanayama \& Pope, 2012). For example, research conducted in the United Kingdom during the 1990s (Korkia \& Stimson, 1993; Lenehan, Bellis, \& McVeigh, 1996) identified categories of users comprising 'competitive sports participants', 'occupational users' and 'aesthetic users' (and a potential fourth group of 'young or novice user')(Dawson, 2001). This work provided a framework based on motivations for anabolic steroid use in the United Kingdom at the time. However, these broad categories of users were unable to capture the complexities of individual ‘drivers' for using AAS (Evans-Brown \& McVeigh, 2008) or specific risk profiles of each of user category.

More recently, Christiansen and colleagues (2016) proposed a typology of four ideal types of AAS users: the 
Expert type; the Athlete type; the Well-being type and the YOLO type. Drawing on international literature and indepth interview data with 37 men, the authors developed a typology that conceptualises variations in AAS use along two dimensions; risk and effectiveness. The resulting typology proposes four ideal 'types' of AAS user, each characterised by a particular set of traits and patterns of engagement with AAS.

The expert type is described as taking controlled risks, they have high levels of knowledge about AAS and associated drugs and are often a source of information and advice for other AAS users. The athlete type primarily use AAS for performance enhancement purposes and are engaged in competitive bodybuilding or sports. The wellbeing type are concerned with looking and feeling good and typically their use of AAS involves low levels of risk (e.g. low/moderate dosages for long term wellbeing). The final group is the YOLO type. YOLO is an acronym for 'You Only Live Once' and this group typifies AAS users who engage with AAS in a 'haphazard' manner and whose use tends to be largely unplanned and driven by the desire to achieve quick improvements in their physique (Christiansen et al., 2016).

Some attention has also been given to examining patterns of IPED use and risk behaviours among subpopulations identified as having a greater propensity to engage in steroid use including young men, adolescents \{Dunn and White, 2011$\}$ \{Mattila, Parkkari, Laakso, Pihlajamaki and Rimpela, 2010\} \{Thorlindsson and Haldorsson, 2010\}, recreational athletes and gym attendees \{Baker, Graham and Davies, $2006\}\{$ Cohen, Collins, Darkes and Gwartney, 2007\}\{Evans, 1997\}, and elite athletes and bodybuilders \{Linqvist, Moberg, Eriksson, 2013$\}$ \{Nogueira, Brito Ade, Oliverira, Veira and Gouveia, 2014\} \{Parkinson and Evans, 2006\}\{Trenton and Currier, 2005\}. Among a sample of adolescent steroid users, Miller and colleagues found inter-group variation in risk taking delineated by gender and athleticism \{Miller et al $2002\}$. Other studies have noted that patterns of steroid use displayed by younger users aged, under 24 years, are associated with higher levels of risks than those exhibited by older steroid users \{Cohen 2007\} \{Chandler and McVeigh 2013\}. 
Understanding different types of AAS use is important for identifying risks and developing targeted policies and interventions. In this study we employ cluster analysis to quantitatively assess AAS user typologies in a sample of 611 AAS using men predominantly recruited through needle and syringe programs and gyms in England and Wales. We then examine variations in motivations for AAS use across different user typologies. The aim of this research is twofold: to establish empirical support for the existence of different types of AAS users and to better understand how motivations for use are associated with different patterns of use and potential for risk. Identification of differing patterns of AAS use by particular subpopulations and how these patterns are related to factors motivating use is important for the development of effective, targeted responses to AAS use.

\section{Methods}

This study draws on data from the 2015 National IPED Info Survey, an annual survey exploring the use of image and performance enhancing drugs conducted in Wales, England and Scotland (Bates \& McVeigh, 2016). Predominantly, recruitment was led by researchers who operated in drug and health services and/or visited gyms and sports settings across 16 locations in Wales, England and Scotland. Researchers approached potential participants in the described settings and established eligibility for survey participation, which was lifetime use of any IPED. Participants were identified through service attendance or within gyms and sport settings through a snowball approach where participants were recommended by other participants and gatekeepers. Snowball referral technique is commonly employed in qualitative research of hard to reach, vulnerable populations, such as people who use illicit drugs. It is important to note that the sampling method employed may have resulted in a sample that is slightly more homogenous than the steroid using population along class, age, sexual orientation and ethnic lines. This risk was minimised by sampling from both gyms and needle and syringe programs across 16 geographic areas. The survey was self-completed either online or in paper form. Ethical approval for the survey was obtained via the Liverpool John Moores University Research Ethics Committee. The survey was open from August-December 2015. The National IPED Info survey collects information on current and previous IPED use, dosages and cycling of IPEDs, injecting 
practices, usual source of IPEDs, experience of adverse effects of IPED use, use of alcohol and other illicit substances in addition to demographics and information on the participant's general health and lifestyle (Bates \& McVeigh, 2016).

\section{Analysis}

\section{Cluster Analysis}

Cluster analysis is shown to be an effective method for identifying groups in data and has been applied to establish typologies of individuals who use cocaine $\{$ Schonnesson. 2008\}; opioids $\{$ Florez , 2015\}, and alcohol $\{$ Peacock, 2016\}. Cluster analysis methodology was applied to our analytic sample using a two-step approach. SPSS two-step clustering uses a log-likelihood distance measure and operates with both continuous and categorical variables. To calculate log-likelihood distance, it is assumed that the continuous variables have normal distributions and the categorical variables have multinomial distributions. The procedure uses an agglomerative hierarchical clustering method. In the first step, hierarchical cluster analysis using Ward's method generates a dendrogram for estimation of the number of likely clusters within the sample. This estimate is pre-specified in a k-means cluster analysis that is used as the principal clustering technique.

Variables chosen for cluster modelling were selected on the basis of their considered contribution to health risks and achieving desired outcomes of steroid use (Christiansen et al., 2016). All measurements were standardized using z scores for continuous variables and 0 or 1 for categorical variables. We included eleven variables in the cluster analysis; 9 categorical and 2 continuous variables. All variables were drawn from the 2015 National IPED survey. A four cluster solution was the best fitting with the following variables: AAS administered orally only (no; yes); engaged in at least one unsafe injecting practice (e.g. sharing equipment, reusing needles) in the previous 12 months (no; yes); used non-AAS IPED in last 12 months ${ }^{1}$ (no; yes); injected peptides in the last 12 months (no; yes); used three or more types oral AAS in the last 12 months (no; yes); used three or more types injectable AAS in the last 12 months (no; yes); frequency of binge

\footnotetext{
1 Used one of DNP; Clenbuterol; Thyroid hormones; Ephedrine; diuretics; Viagra; prohormones in addition to AAS.
} 
drinking $^{2}$ (never; less than monthly; monthly; weekly; daily or almost daily); frequency of alcohol consumption (never; monthly or less; 2-4 times per month; 2-3 times per month; 4 or more times a week); usual IPED source (friend; dealer; trainer; internet; underground lab; bought abroad; home made; prescribed by a doctor; multiple sources); total number of self-reported physiological and psychological adverse effects in the last 12 months (continuous); number of recreational drug types used in the last 12 months (continuous). Discriminant function modelling identified the majority of parameters used in the cluster analysis to be significant determinants of cluster membership. The average measure of cohesion and separation was 0.22 indicating that the within-cluster differences were smaller than between cluster differences. The silhouette measure ranges from -1 to +1 with values closer to +1 indicating better fit.

\section{Multinomial logistic regression}

Following the cluster analysis, we use multinomial logistic regression to examine whether AAS user typology, is predicted by current age; age first used AAS and/or motivations for using AAS. We use cluster group 2 as the reference category as it is the largest group.

\section{Results}

The 2015 National IPED Info Survey comprised a total of 663 participants. The final analytic cohort for this study comprised male participants only for whom a full composite of relevant variables were available $(n=611)$. The average age of the sample was 30.5 years $(s d=8.8)$ and the majority of the sample were White British ( $\mathrm{n}=437,71.5 \%)$. Summary statistics for all variables are provided in Table 1.

\section{Cluster Analysis}

The cluster analysis identified four groups in the data (see Table 1). Cluster one contains 68 (11.1\%) individuals with an average age of 26.4 years $(\mathrm{SD}=9.0)$. On average, individuals in cluster one are younger than those in other groups. Two other key features define this cluster; individuals in this group tend to use oral AAS only (76.0\%) and they report higher levels of alcohol use than individuals in other clusters. More

\footnotetext{
2 Here binge drinking is defined as 8 or more units of alcohol on a single occasion.
} 
than half of the individuals in this group (54.6\%) report drinking alcohol 2-4 times a month and one third (32.8\%) report binge drinking weekly. On average individuals in this group report using one psychoactive drug in the last 12 months. Individuals in this cluster are the least likely to report using peptides and only a quarter report using other IPEDs in addition to AAS. On average, they reported fewer adverse effects than individuals in other groups. Most commonly, individuals in cluster 1 reported sourcing AAS through friends (41.8\%). This cluster can be seen to represent the YOLO type.

Cluster 2 contains $236(38.6 \%)$ individuals with an average age of 30.6 years $(\mathrm{sd}=8.3)$. This cluster can be seen as representing the average AAS user in the sample. Individuals in cluster 2 are unlikely to use other IPEDs in addition to AAS (2.9\%) and the majority have used two or less injectable AAS in the past 12 months $(12.3 \%)$. The majority of individuals in cluster 2 report drinking alcohol either, $2-4$ times per month $(45.8 \%)$, or monthly or less $(38.6 \%)$. Most commonly, individuals in this group report binge drinking less than monthly (36.4\%) and on average report using fewer psychoactive drugs than clusters 1 and 3 . On average, cluster 2 individuals have experienced 1.1 adverse effects related to their AAS use and most commonly report sourcing their AAS through friends (37.3\%). This cluster can be seen to represent the Wellbeing type.

Cluster 3 contains $155(25.4 \%)$ individuals with an average age of 32.2 years ( $\mathrm{sd}=9.1)$. Individuals in this cluster can be differentiated from individuals in the other three clusters by their use of peptides and other IPEDs in addition to AAS. Additionally, individuals in this cluster are more likely than those in clusters 1, 2 and 4 to have used three or more types of oral and injectable AAS during the last 12 months. On average, individuals in cluster three use more psychoactive substances (mean=1.5, $\mathrm{sd}=1.7$ ) and experience more side effects (mean=3.4, $\mathrm{sd}=2.5$ ) than individuals in the other clusters. It is important to note that the data does not contain information on whether psychoactive drug use was primarily recreational or instrumental. This group of substances includes those that are noted accompaniments to steroids among athletes. Psychoactive substances taken for performance enhancement include: stimulants, anti-depressants and tranquilisers to aid sleep and recovery and opioids for pain management \{Miller 2002\}\{Sagoe 2015\}. Most commonly, 
individuals in cluster 3 acquire IPEDs from multiple sources (32.9\%); more than a quarter (27.7\%) report sourcing their IPEDs through a dealer. This cluster can be seen to represent the Athlete typology.

Cluster 4 contains $152(24.9 \%)$ of individuals with an average age of 30.6 years $(\mathrm{sd}=8.7)$. The defining characteristic of cluster 4 is patterns of alcohol use; $88.7 \%$ of individuals in this group never consume alcohol and 100\% report that they never binge drink. On average individuals in cluster 4 report using fewer psychoactive drugs than individuals in clusters 1,2 or 3 . Individuals in this group are unlikely to report that they use IPEDs other than AAS and only a quarter report using three or more AAS in the last 12 months (25.7\%). On average this group have experienced $1.3(\mathrm{sd}=1.7)$ adverse effects and most commonly report acquiring AAS through multiple sources (34.2\%). This cluster can be seen to represent the Expert typology.

>> Table 1 here $<<<$

\section{Multinomial Logistic Regression}

The results of the multinomial logistic regression are summarised in Figure 1 and displayed in Table 2. As cluster 2 was the largest category and was representative of the average AAS user in the sample we use it as the reference category in the multinomial logistic regression. Results of a multinomial logistic regression demonstrate, individuals are more likely to be in cluster 1 (YOLO) than in cluster 2 (Wellbeing), if they are younger $(B=-0.05, p<0.1)$, started using AAS at a younger age $(B=-0.06, p<0.1)$ and are motivated to use AAS to lose fat $(\mathrm{B}=0.19, \mathrm{p}<0.01)$. Individuals are more likely to be in cluster 3 (Athlete) than in cluster 2 (Wellbeing) if they are older $(\mathrm{B}=0.06, \mathrm{p}<0.001)$; started using AAS at a younger age $(\mathrm{B}=-0.08, \mathrm{p}<0.001)$ and do not self-identify as 'white British' $(B=0.99, \mathrm{p}<0.01)$. Individuals in cluster 3 are motivated to use AAS to gain muscle $(B=2.68, p<0.05)$ and lose fat $(B=0.61, p<0.05)$. Finally, individuals are more likely to be in cluster 4 (Expert) than cluster 2 (Wellbeing) if they do not self-identify as white British $(\mathrm{B}=0.60, \mathrm{p}<0.05)$ and if they are not motivated by fitness goals $(B=-0.71, p<0.05)$.

$>>>$ Figure 1 here $<<<$ 


\section{Discussion}

Taken together the results of the cluster analysis and logistic regression provide support for the existence of AAS subpopulations that differ in regards to patterns of use and motivations for use. The groups defined through our cluster analysis are broadly consistent with the four ideal types proposed by Christiansen and colleagues (2016). We suggest that clusters 1,2, 3 and 4 broadly correspond with the YOLO, Wellbeing, Athlete and Expert types respectively. However, we also note important differences between our results and Christiansen et al's \{Christiansen 2016\} typology; we outline similarities and differences below.

Most of the characteristics of cluster 1 align with those of the YOLO type; young, inexperienced, high levels of lifestyle risk (i.e. highest alcohol consumption). Cluster 1 individuals' tendency to use only oral AAS and to source these drugs through friends also fits with the depiction of the YOLO user who is influenced by peers, inexperienced, peripheral to the bodybuilding or competitive sports fields and less likely to see effective outcomes of their use. The tendency for individuals in cluster 1 to use oral steroids sourced from a friend may indicate experimental or unplanned use. It may also reflect this groups lower knowledge of steroids and fewer connections that provide access to injecting equipment and information on preparation and injection techniques. Use in this group was motivated by fat loss, which also speaks to the imagefocussed goals implied in the YOLO typology. There are two notable departures from Christiansen et al's $\{$ Christiansen 2016\} description of the YOLO type. The first is that cluster 1 individuals report experiencing very few adverse effects. This finding is likely related to the relative youth of cluster 1 and their tendency to engage in oral steroid use; the majority of adverse effects reported by participants from clusters 2 to 4 were related to injecting. Further, unlike injecting injuries that are immediately visible, adverse effects specific to oral steroid use, such as impacts on the liver and kidneys require diagnosis by a health care professional. Therefore, individuals may be unaware of adverse effects that they have experienced. The second point of difference between cluster 1 and the YOLO type is low levels of psychoactive drug use reported by cluster 1 compared to clusters 2 to 4 . We suggest this outcome may reflect sampling sites; Cluster 1 individuals typically used only oral steroids and were sampled from gyms (did not attend NSPs). Therefore, we may 
expect the other cluster types, containing individuals sampled from both gyms and NSPs to report higher levels of psychoactive drug use.

Cluster 2 was the largest of our clusters and was characteristic of the Wellbeing type. The wellbeing type described by Christianson and colleagues (2016) represents the majority of gym going users who are concerned with looking and feeling good and achieving a healthy body. This group avoid risk by taking a long-term, lifestyle approach to using AAS. Similarly, we found that cluster 2 individuals engaged in low risk AAS use with few using other IPEDs and/or more than three types of AAS. This group consumed alcohol at normal population levels suggesting that AAS use is part of a lifestyle rather than part of a wider regime involving strict diet such is the case with the Athlete type. This group were more likely than the expert group to be motivated by 'getting fit', which corresponds with the Wellbeing group outlined by Christianson and colleagues (2016).

Individuals in cluster 3 represent the Athlete type. Christiansen et al (Christiansen et al., 2016) describe the Athlete type as primarily engaging in AAS use to prepare for bodybuilding competitions or strength events. For these individuals ASS use, along with associated risks, are a necessary part of the competition preparations. This type of user may engage in risky patterns of use but their use is also highly effective in achieving strength and muscle gains. Similar to the Athlete type, individuals in cluster 3 tended to use a greater number of AAS and IPEDs. Also in line with the Athlete type, cluster 3 individuals reported low levels of alcohol consumption. One of the characteristics of cluster 3 that does not align with the Athlete type is the high rate of psychoactive drug use among this group. However, as we were unable to discern the purpose of psychoactive drug use from our data we cannot determine whether or not the use of psychoactive substances was primarily motivated by recreational or instrumental goals. Instrumental use of psychoactive substances has been noted among bodybuilding samples and may be occurring among this group (Sagoe et al., 2015). Instrumental use refers to use that is based on the effects of the drug for a specific purpose. For example, stimulants such as amphetamine may be used to facilitate training, enhance fat loss and suppress appetite (Sagoe et al., 2015). The Athlete type are noted for combining steroids with other enhancement 
drugs (polypharmacy), therefore instrumental use of psychoactive drugs would be in line with the profile. While all clusters were motivated by muscle gain and fat loss to some degree, a far higher proportion of cluster three were motivated by these goals than other clusters; which aligns with the goals of the competitive bodybuilder.

Characteristics of cluster 4 align with the Expert type. The Expert type engage in low risk AAS use and are well informed on the science of effective AAS use. Cluster 4 is characteristic of the Expert type. Individuals in cluster 4 tend to use fewer than 3 types of AAS and patterns of use of other substances supports effective AAS outcomes; that is, they do not drink alcohol and take few psychoactive substances. Cluster 4 are the less likely, when compared to cluster 2 (Wellbeing) to state achieving 'fitness' as a motivating factor for their AAS use. Being that the expert type is fascinated with the effects of AAS on human psychology and is well versed in the scientific evidence regarding the effects of AAS on the body it is unlikely that they would associate AAS use with 'fitness'; AAS use is not associated with increased cardiovascular conditioning.

An understanding of the types of AAS use, and their associated motivations, risk behaviours and vulnerabilities, is important for the development and provision of appropriate policy responses. Understanding the types of AAS use is also key to the development and implementation of effective interventions, across the spectrum of drug prevention, harm reduction, treatment, rehabilitation and relapse prevention, for this group. While, a range of effective tools have been developed, to influence both the initiation of drug use and its potential escalation, evidence relating to the use of AAS in the general population is lacking (United Nations on Drugs and Crime, 2015). Building on the earlier work of Lenehan, Bellis \& McVeigh (1996) and Dawson (2001) and developing quantitative typologies that compare to those formulated by Christiansen, Vinther, \& Liokaftos (2016) we present a nuanced picture of sub-populations of AAS users. Combined with growing evidence bases related to health risks (Pope et al., 2014) and concomitant behavioural risks including sex, poly-drug use and injecting practices, these typologies can be used to inform targeted harm minimisation approaches to steroid use. 
While the results of this study are informative there are several limitations that must be noted. The original typologies described by Christiansen and colleagues (2016) consider two dimensions of AAS use, perceived risk and effectiveness. Here we include variables to capture risk however we do not have data to measure effectiveness, in part because this is difficult to assess through a short, cross-sectional survey. Further, as the sample was recruited through gyms and NSPs, we may have under and/or over recruited from some groups and some of the types of AAS users in the general population. In particular, YOLO users may be underrepresented in this sample as they tended to use oral steroids only so were only recruited from gym sites.

\section{Conclusion}

This paper illustrates the heterogeneous nature of anabolic steroid use. As is the case with psychoactive drug use, profiles of use and the associated risks are highly variable. This study has contributed to the literature by developing a quantitative typology of AAS users which, in turn, should assist in the formulation of effective demand reduction strategies coupled with targeted harm reduction interventions. However, while such generalist classifications may be useful for interventions at a population level, the data also indicate a highly diverse population of users, highlighting the need for individualised care, based on thorough assessment of risk behaviour, lifestyle and underlying health. Conceptualisation of the steroid user typology is still at the early stages of development. Analysis of future United Kingdom National IPED Survey data together with a range of other diverse datasets will enable this model to be refined, including the acknowledgement of variations within groups and exploring the potential for alternative typologies reflecting recruitment from other settings and/or other countries. 


\section{References}

Bates, G., \& McVeigh, J. (2016). Image and Performance Enhancing Drugs- 2015 Survey Results. Retrieved from Liverpool: http://www.cph.org.uk/wp-content/uploads/2016/07/IPEDs-2015survey final1.pdf

Christiansen, A. V., Vinther, A. S., \& Liokaftos, D. (2016). Outline of a typology of men's use of anabolic androgenic steroids in fitness and strength training environments*. Drugs: education, prevention and policy, 1-11. doi:10.1080/09687637.2016.1231173

Dawson, R. T. (2001). Drugs in sport-The role of the physician. J Endocrinol, 170. doi:10.1677/joe. 0.1700055

Evans-Brown, M. , Kimergård, A., \& McVeigh, J. (2009). Elephant in the room? The methodological implications for public health research of performance-enhancing drugs derived from the illicit market. Drug Test Anal, 1. doi:10.1002/dta.74

Evans-Brown, M., \& McVeigh, J. (2008). Anabolic steroid use in the general population of the United Kingdom. In V. Moller, P. Dimeo, \& M. McNamee (Eds.), Elite sport, doping, and public health. Odense: University of Southern Denmark Press.

Hakansson, A., Mickelsson, K., Wallin, C., \& Berglund, M. (2012). Anabolic Androgenic Steroids in the General Population: User Characteristics and Associations with Substance Use. European Addiction Research, 18(2), 83-90. doi:10.1159/000333037

Hanley Santos, G., \& Coomber, R. (2017). The risk environment of anabolic-androgenic steroid users in the UK: Examining motivations, practices and accounts of use. International Journal of Drug Policy, 40, 35-43. doi:http://dx.doi.org/10.1016/j.drugpo.2016.11.005

Kanayama, G., \& Pope, H. G. (2012). Illicit Use of Androgens and Other Hormones: Recent Advances. Current Opinion in Endocrinology, Diabetes, and Obesity, 19(3), 211-219. doi:10.1097/MED. Ob013e3283524008

Kanayama, G., \& Pope Jr, H. G. (2017). History and epidemiology of anabolic androgens in athletes and non-athletes. Molecular and Cellular Endocrinology. doi:http://dx.doi.org/10.1016/j.mce. 2017.02.039

Korkia, P., \& Stimson, G. (1993). Anabolic steroid use in Great Britain: an exploratory investigation. Final report to the Department of Health for England, Scotland and Wales. London: The Centre for Research on Drugs and Health Behaviour.

Lenehan, P., Bellis, M., \& McVeigh, J. (1996). A study of anabolic steroid use in the North West of England. J Perform Enhanc Drugs, 1.

McVeigh, J., Evans-Brown, M., \& Bellis, M. (2012). Drogas potenciadoras para la búsqueda de la perfección. Adicciones, 24(3), 185-190.

Pope, J. H. G., Wood, R. I., Rogol, A., Nyberg, F., Bowers, L., \& Bhasin, S. (2014). Adverse Health Consequences of Performance-Enhancing Drugs: An Endocrine Society Scientific Statement. Endocrine Reviews, 35(3), 341-375. doi:10.1210/er.2013-1058

Sagoe, D., McVeigh, J., Bjørnebekk, A., Essilfie, M.-S., Andreassen, C. S., \& Pallesen, S. (2015). Polypharmacy among anabolic-androgenic steroid users: a descriptive metasynthesis. Substance Abuse Treatment, Prevention, and Policy, 10(1), 12. doi:10.1186/s13011-015-0006-5

United Nations on Drugs and Crime. (2015). International Standards on Drug Use Prevention. Retrieved from Vienna: 
Figure 1. Features of Christiansen et al. (2016) steroid user typologies and the four clusters

\section{The YOLO type}

1. Motivated by impatience, curiosity and influence from peers and authoritative role models

2. Focus on asserting masculinity, impressing girls, advancing upwards in the male hierarchy

3. Little to no concern about side effects

4. Aims at immediate benefits

5. Low level of knowledge- based on lore and advice from gym 'rats'

6. Hedonistic and 'flying blind' attitude towards health-combines AAS with recreational drugs and is more likely to end up in fights than the other types

7. Little concern about diet and recovery has an experimenting life style which includes various types of risk taking.

\section{Cluster 1}

A. Younger age group on average than other clusters

B. Typically use oral AAS and rarely use other IPEDs

C. High levels of alcohol use including binge drinking

D. Experienced few adverse effects

\section{The Athlete type}

1. Motivated by competitive aspirations

2. Focus on performance (e.g. skills, size and definition)

3. Concerned with side effects but willing to run health risks to fulfil sporting ambitions

4. Aims at maximising benefits by combining different steroids (stacking) with other performance enhancing drugs (polypharmacy)

5. Medium to high level of knowledge - based on medical assistance and experience from his sporting community

6. Plans training and diet according to season-avoids recreational drugs

7. Dedicated to the athlete lifestyle

\section{Cluster 3}

A. Use a range of oral and injectable AAS

B. Use a range of IPEDs in addition to AAS

C. Moderate to low levels of alcohol consumption but greater psychoactive drug use than other clusters

D. Experience more adverse effects

\section{The Well-being type}

1. Motivated by vanity and/wishes for restoration or rejuvenation

2. Focus on well-being, moderation and peer recognition

3. Considers side effects and wants to play it safe

4. Aims at slight improvements

5. Medium level of knowledge- based on own experience, online fora and other users

6. Wants to improve his quality of life and has relaxed take on nutrition

7. Focus on healthy living and are typically older than other types

\section{Cluster 2}

A. Unlikely to use other IPEDs in addition to AAS

B. Typically use few types of AAS

C. Moderate levels of alcohol consumption and psychoactive drug use

D. Experience few adverse effects

\section{The Expert type}

1. Motivated by scientific curiosity and fascination with pharmacological performance enhancement

2. Focus on muscularity, learning, information sharing

3. Concerned with side effects, wants to play it safe

4. Aims at optimising benefits with a perceived sensible drug regimen

5. High level of knowledge - based on various sources including scientific papers

6. Values health and monitors his body systematically

7. Offers advice on harm reduction to others and seeks recognition as an expert on AAS

\section{Cluster 4}

A. Typically use few types of AAS

B. Moderate use of peptides and other IPEDs in addition to AAS

C. Rarely drink alcohol or use psychoactive drugs

D. Experience few adverse effects 
Table 1. Summary statistics

\begin{tabular}{|c|c|c|c|c|c|}
\hline & $\begin{array}{l}\text { Sample } \\
(\mathrm{n}=611 ; 100 \%)\end{array}$ & $\begin{array}{l}\text { Cluster } 1 \quad(n=68 \\
11.1 \%)\end{array}$ & $\begin{array}{l}\text { Cluster } 2 \\
(\mathrm{n}=236 ; 38.6 \%)\end{array}$ & $\begin{array}{l}\text { Cluster } 3 \\
(\mathrm{n}=155 ; 25.4 \%)\end{array}$ & $\begin{array}{l}\text { Cluster } 4 \\
(\mathrm{n}=152 ; 24.9 \%)\end{array}$ \\
\hline Current age: mean (sd) & $30.54(8.81)$ & $26.37(8.95)$ & $30.62(8.32)$ & $32.15(9.05)$ & $30.64(8.74)$ \\
\hline Age first use: mean (sd) & $24.39(6.54)$ & $22.21(5.14)$ & $25.37(7.08)$ & $23.70(6.20)$ & $24.56(6.30)$ \\
\hline Ethnicity: non-White British & $174(28.28 \%)$ & $16(23.53 \%)$ & $47(19.92 \%)$ & $65(41.94 \%)$ & $46(30.26 \%)$ \\
\hline \multicolumn{6}{|l|}{ Steroid Use } \\
\hline Oral steroids only (never injected) & $62(10.15 \%)$ & $60(75.95 \%)$ & $1(0.42 \%)$ & $0(0.00 \%)$ & $1(0.66 \%)$ \\
\hline $\begin{array}{l}\text { Unsafe injecting practices in the last } \\
12 \text { months }\end{array}$ & $529(86.58 \%)$ & $0(0.00 \%)$ & $234(99.15 \%)$ & $148(95.48 \%)$ & $147(96.71 \%)$ \\
\hline Used three or more oral AAS & $56(9.17 \%)$ & $4(5.88 \%)$ & $0(0.00 \%)$ & $48(30.97 \%)$ & $4(2.63 \%)$ \\
\hline Used three or more injectable AAS & $204(33.39 \%)$ & $1(2.94 \%)$ & $29(12.29 \%)$ & $134(86.45 \%)$ & $39(25.66 \%)$ \\
\hline $\begin{array}{l}\text { Used IPEDs in addition to AAS in } \\
\text { the last } 12 \mathrm{mths}\end{array}$ & $154(25.20 \%)$ & $17(25.00 \%)$ & $7(2.97 \%)$ & $102(65.81 \%)$ & $28(18.42 \%)$ \\
\hline Injected peptides in the last $12 \mathrm{mths}$ & $195(31.91 \%)$ & $0(0.00 \%)$ & $56(23.73 \%)$ & $87(56.13 \%)$ & $52(34.21 \%)$ \\
\hline \multicolumn{6}{|l|}{ IPED source } \\
\hline Friend & $189(30.93 \%)$ & $28(41.18 \%)$ & $88(37.29 \%)$ & $40(25.81 \%)$ & $33(21.71 \%)$ \\
\hline Dealer & $135(22.09 \%)$ & $13(19.12 \%)$ & $40(16.95 \%)$ & $43(27.74 \%)$ & $39(25.66 \%)$ \\
\hline Trainer & $22(3.60 \%)$ & $2(2.94 \%)$ & $14(5.93 \%)$ & $3(1.94 \%)$ & $3(1.97 \%)$ \\
\hline Internet & $68(11.13 \%)$ & $7(10.29 \%)$ & $33(13.98 \%)$ & $10(6.45 \%)$ & $18(11.84 \%)$ \\
\hline Underground lab & $15(2.45 \%)$ & $1(1.47 \%)$ & $5(2.12 \%)$ & $6(3.87 \%)$ & $3(1.97 \%)$ \\
\hline Bought abroad & $13(2.13 \%)$ & $0(0.00 \%)$ & $7(2.97 \%)$ & $2(1.29 \%)$ & $4(2.63 \%)$ \\
\hline Multiple sources & $169(27.66 \%)$ & $17(25.00 \%)$ & $49(20.76 \%)$ & $51(32.90 \%)$ & $52(34.21 \%)$ \\
\hline $\begin{array}{l}\text { Number of adverse effects in the last } \\
12 \text { mths (max.10): mean (sd) }\end{array}$ & $1.69(2.0)$ & $0.78(1.03)$ & $1.07(1.28)$ & $3.39(2.47)$ & $1.32(1.67)$ \\
\hline
\end{tabular}

\section{Lifestyle}

Frequency alcohol consumption

Never
Monthly or less
2-4 times a month
4 times a week

$\begin{array}{lllll}164(27.06 \%) & 13(19.70 \%) & 0(0.00 \%) & 17(11.11 \%) & 134(88.74 \%) \\ 207(34.16 \%) & 13(19.70 \%) & 91(38.56 \%) & 89(58.17 \%) & 14(9.27 \%) \\ 186(30.69 \%) & 36(54.55 \%) & 108(45.76 \%) & 39(25.49 \%) & 3(1.99 \%) \\ 27(4.46 \%) & 4(6.06 \%) & 19(8.05 \%) & 4(2.61 \%) & 0(0.00 \%) \\ 22(3.63 \%) & 0(0.00 \%) & 18(7.63 \%) & 4(2.61 \%) & 0(0.00 \%)\end{array}$

Frequency of binge drinking

Never
Less than monthly
Monthly
Weekly
Daily (almost daily)

Number of psychoactive drugs used in the last 12 months (max. 11): mean (sd)

$\begin{array}{lllll}201(33.61 \%) & 13(20.31 \%) & 8(3.39 \%) & 30(20.27 \%) & 150(100.00 \%) \\ 176(29.43 \%) & 15(23.44 \%) & 86(36.44 \%) & 75(50.68 \%) & 0(0.00 \%) \\ 113(18.90 \%) & 15(23.44 \%) & 73(30.93 \%) & 25(16.89 \%) & 0(0.00 \%) \\ 102(17.06 \%) & 21(32.81 \%) & 63(26.69 \%) & 18(12.16 \%) & 0(0.00 \%) \\ 6(1.00 \%) & 0(0.00 \%) & 6(2.54 \%) & 0(0.00 \%) & 0(0.00 \%) \\ 0.83(1.40) & 0.91(1.81) & 0.67(1.18) & 1.48(1.73) & 0.41(0.78)\end{array}$

\section{Motivations}




$\begin{array}{llllll}\text { Gain muscle } & 554(90.67 \%) & 56(82.35 \%) & 211(89.41 \%) & 154(99.35 \%) & 133(87.50 \%) \\ \text { Lose fat } & 294(48.12 \%) & 29(42.65 \%) & 103(43.64 \%) & 97(62.58 \%) & 65(42.76 \%) \\ \text { Get stronger } & 402(65.79 \%) & 30(44.12 \%) & 154(65.25 \%) & 118(76.13 \%) & 100(65.79 \%) \\ \text { Get fitter } & 192(31.42 \%) & 20(29.41 \%) & 82(34.75 \%) & 53(34.19 \%) & 37(24.34 \%) \\ \text { Get faster } & 85(13.91 \%) & 10(14.71 \%) & 30(12.71 \%) & 26(16.77 \%) & 19(12.50 \%) \\ \text { Improve endurance } & 133(21.77 \%) & 9(13.24 \%) & 52(22.03 \%) & 39(25.16 \%) & 33(21.71 \%) \\ \text { Increase sex drive } & 54(8.84 \%) & 1(1.47 \%) & 18(7.63 \%) & 26(16.77 \%) & 9(5.92 \%)\end{array}$


Table 2. Multinomial logistic regression: reference category Cluster $2(\mathrm{n}=611)$

\begin{tabular}{|c|c|c|c|c|c|c|c|c|c|}
\hline & \multicolumn{3}{|c|}{ Cluster 1} & \multicolumn{3}{|c|}{ Cluster 3} & \multicolumn{3}{|c|}{ Cluster 4} \\
\hline & $\mathrm{B}(\mathrm{sd})$ & RRR & $\mathrm{p}$ & $\mathrm{B}(\mathrm{sd})$ & RRR & $\mathrm{p}$ & $\mathrm{B}(\mathrm{sd})$ & RRR & $\mathrm{p}$ \\
\hline Current age & $-0.05(0.03)$ & 0.95 & $\wedge$ & $0.06(0.02)$ & 1.03 & $* * *$ & $0.02(0.02)$ & 1.02 & \\
\hline Age first use & $-0.06(0.04)$ & 0.94 & $\wedge$ & $-0.08(0.02)$ & 0.92 & $* * *$ & $-0.04(0.02)$ & 0.96 & $\wedge$ \\
\hline Non-white British & $0.26(0.35)$ & 1.30 & & $0.99(0.25)$ & 2.70 & $* * *$ & $0.60(0.25)$ & 1.82 & * \\
\hline \multicolumn{10}{|l|}{ Motivations } \\
\hline Gain muscle & $-0.65(0.42)$ & 0.52 & & $2.68(1.03)$ & 14.66 & $*$ & $-0.31(0.35)$ & 0.74 & \\
\hline Lose fat & $0.19(0.31)$ & 1.21 & $* *$ & $0.61(0.24)$ & 1.84 & $*$ & $0.04(0.23)$ & 1.04 & \\
\hline Get stronger & $-1.07(0.33)$ & 0.34 & & $0.43(0.27)$ & 1.54 & & $0.23(0.25)$ & 1.26 & \\
\hline Get fitter & $0.16(0.38)$ & 1.17 & & $-0.43(0.28)$ & 0.65 & & $-0.71(0.28)$ & 0.49 & * \\
\hline Get faster & $0.49(0.47)$ & 1.64 & & $0.02(0.37)$ & 1.02 & & $0.26(0.37)$ & 1.30 & \\
\hline Improve endurance & $-0.45(0.45)$ & 0.64 & & $-0.03(0.30)$ & 0.97 & & $0.15(0.29)$ & 1.17 & \\
\hline Increase sex drive & $-1.65(1.08)$ & 0.19 & & $0.50(0.38)$ & 1.65 & & $-0.46(0.45)$ & 0.63 & \\
\hline Constant & $2.64(0.83)$ & 14.01 & $* *$ & $-3.66(1.16)$ & 0.03 & $* *$ & $-0.07(0.59)$ & 0.94 & \\
\hline Log likelihood & \multicolumn{9}{|c|}{-725.73} \\
\hline $\operatorname{LR~chi}^{2}(30)$ & \multicolumn{9}{|c|}{$144.30^{* * *}$} \\
\hline Pseudo R² & \multicolumn{9}{|c|}{$9.04 \%$} \\
\hline
\end{tabular}

NOTES: ${ }^{\wedge} \mathrm{p}<0.1 ;{ }^{*} \mathrm{p}<0.05 ;{ }^{* *} \mathrm{p}<0.01 ; * * * \mathrm{p}<0.001$ 